\title{
Happiness and depression in psoriasis: a cross-sectional study in Germany
}

\author{
Barbara Schuster ${ }^{1,2}$ (1) $\cdot$ Corinna Peifer $^{3} \cdot$ Stefanie Ziehfreund $^{1} \cdot$ Linda Tizek $^{1,2} \cdot$ Tilo Biedermann $^{1} \cdot$ Alexander Zink $^{1,4}$. \\ Maximilian C. Schielein ${ }^{1,2,4}$
}

Accepted: 31 August 2021 / Published online: 16 September 2021

(c) The Author(s) 2021

\begin{abstract}
Purpose Prior research on the psychological consequences of skin diseases has focused on assessing mental comorbidities. The aim of this study was to investigate subjective well-being in a large sample of individuals affected by psoriasis, a chronic inflammatory skin disease, and to explore the associations with depression and disease-related parameters such as disease severity.

Methods A cross-sectional online survey was conducted from March to June 2019. The link to the questionnaire was shared on websites and Facebook pages of psoriasis patient organizations and campaigns. Participants filled in validated scales measuring subjective well-being-operationalized as positive affect (PA), negative affect (NA) and satisfaction with life (SWL); and depression.

Results The data of 722 participants were analyzed. Exploratory factor analysis supported the differentiation of PA, NA, SWL, and depression as four different constructs. The respondents reported lower levels of PA than healthy individuals and judged themselves to be less happy and were less satisfied with their lives than the general population (except age group $65+$ years). $40.3 \%$ of respondents were screened positive for depression. More severe psoriasis was associated with lower affective well-being and a higher risk for depression.

Conclusion The results of this study empirically supported the differentiation of subjective well-being and depression as different constructs in individuals with psoriasis, and underline the large mental burden of the disease which goes beyond a higher risk for depression. Measures of well-being should thus be incorporated in both research and clinical practice in patients with psoriasis in order to achieve a more comprehensive picture of the mental burden of this disease.
\end{abstract}

Keywords Psoriasis · Happiness $\cdot$ Subjective well-being $\cdot$ Depression $\cdot$ Mental health $\cdot$ Dermatology

\section{Plain English summary}

Alexander Zink and Maximilian C. Schielein have contributed equally to this work.

Barbara Schuster

barbara.schuster@tum.de

1 Department of Dermatology and Allergy, School of Medicine, Technical University of Munich, Munich, Germany

2 Institute for Medical Information Processing, Biometry, and Epidemiology (IBE), Pettenkofer School of Public Health, LMU Munich, Munich, Germany

3 Department of Psychology, University of Lübeck, Lübeck, Germany

4 Division of Dermatology and Venereology, Department of Medicine, Karolinska Institutet, Stockholm, Sweden
Chronic skin diseases such as psoriasis can be a large mental burden for the affected individuals. Previous research has focused on assessing mental comorbidities such as depression in order to determine mental health in patients with skin diseases. However, according to the World Health Organization, there is more to being well than just not being ill. Thus, in order to fully capture the mental burden of skin diseases, both mental comorbidities and well-being need to be considered. Therefore, the aim of this study was to assess the mental burden of patients with psoriasis by not only measuring depression, but also happiness. We found that patients with psoriasis are less happy and have a higher risk for depression than healthy individuals. Treating skin symptoms can help the patients to be happier and less depressed. 
However, additional psychological care should be provided to patients with severe psoriasis in order to improve the patients' well-being.

\section{Introduction}

Psoriasis is a common chronic inflammatory skin disease with an estimated prevalence of $1.2-3 \%$ in Germany [1, 2]. It is characterized by sharply demarcated red patches covered in typical whitish-silver scales, typically, but not only, located on knees and elbows as well as the scalp [3]. A large number of patients suffer from severe itch, and some of them, even pain [4, 5]. In addition, psoriasis is associated with a large number of comorbidities, ranging from arthritis and metabolic syndrome to depression $[3,6,7]$. As psoriatic lesions are often considered disfiguring by their social environments, many affected patients experience body image issues [8] and suffer from stigmatization and social exclusion $[9,10]$, resulting in a large psychological disease burden [7, $11,12]$. As a consequence, psoriasis has been recognized as a serious noncommunicable disease by the World Health Assembly in 2014 [13].

\section{Assessing the mental burden of psoriasis}

Prior research on the psychological consequences of skin diseases has predominantly taken a pathogenic perspective, with most studies exploring mental comorbidities as markers of mental health. The results in general indicated a higher risk of depression, anxiety, and addiction for patients with psoriasis [6, 14-16]. However, in line with the World Health Organization (WHO) defining health as "a state of [...] wellbeing and not merely the absence of disease" [17], salutogenic approaches for measuring mental health in patients with skin diseases are necessary in order to achieve a holistic understanding of the mental burden [12, 18, 19]. Accordingly, over the last years, studies exploring well-being in individuals with psoriasis have emerged [20-24]. In general, most studies comparing individuals with psoriasis to healthy controls found that well-being was impaired in this patient group, underlining the large mental burden of psoriasis [20, $21,25]$. However, there is increasing evidence pointing toward a more complex relationship between psoriasis and well-being, which seems to be mediated by the subjective perception of the symptoms rather than the objective course of the disease [21, 26, 27].

\section{Subjective well-being and depression in psoriasis}

Recently, happiness has been discussed as a patient reported outcome with high subjective relevance for patients with chronic skin diseases [20]. Although different definitions exist [28], happiness is often conceptualized as subjective well-being [29]. According to Diener et al. [25], subjective well-being comprises an individual's "cognitive and affective evaluation of life as a whole". Following this approach, subjective well-being is high if one experiences frequent positive affect (PA), infrequent negative affect (NA) and a general satisfaction with life (SWL) $[25,30]$. So far, only a few studies exploring subjective well-being in patients with psoriasis exist [23, 24, 31, 32], with only one study comprehensively assessing all three facets of subjective well-being in a comparably small group of 52 patients with psoriasis [20]. The results of the existing studies suggest that psoriasis patients are impaired in terms of PA but not SWL compared to healthy individuals [20, 23, 24]. For NA, results are inconsistent [20, 23, 31].

Depression, in contrast, is a well-researched and generally accepted comorbidity of psoriasis which has been studied in several systematic reviews and meta-analyses [33, 34]. As depression is characterized by the reduced capability to experience positive emotions [35], the finding of low PA in patients with psoriasis could be explained by the higher prevalence of depressive symptoms in this patient group, which raises the question whether exploring affective wellbeing independently of depression does in fact provide new insights on the mental burden of psoriasis. Studies exploring both subjective well-being and depression in individuals with psoriasis are needed in order to be able to distinguish these two constructs in this patient group.

\section{Aim of the study}

The aim of this study was thus to assess both depression and subjective well-being (operationalized as PA, NA, and SWL) in a large and diverse sample of individuals affected by psoriasis, and to explore associations between depression, subjective well-being, and patient characteristics in this group.

\section{Materials and methods}

\section{Study population and recruitment}

For this cross-sectional study, an online survey among individuals affected by psoriasis in Germany was conducted from March to June 2019. The link to the online questionnaire was shared on Germany's largest patients' organization webpage for psoriasis ("Psoriasis Netz", www.psori asis-netz.de) and in the corresponding email newsletter and forum. In addition, the link was shared on other psoriasisrelated Facebook pages ("Farbenhaut", Technical University of Munich's University Hospital and the de-stigmatization campaign "Bitte berühren" by the Professional Association of the German Dermatologists). Only individuals affected 
by psoriasis were eligible to participate, which was clearly stated on the first page of the online questionnaire and which had to be confirmed by the participants in the first question before they were able to continue with the rest of the questionnaire. Informed consent was obtained from all participants prior to inclusion. This study was conducted in accordance with the Declaration of Helsinki and was reviewed and approved by the local ethics committee of Technical University of Munich (reference 25/19 S).

\section{Study variables}

The study questionnaire was developed by a multidisciplinary team including a dermatologist, a psychologist and two epidemiologists. It consisted of validated scales measuring subjective well-being and depression and additional questions on happiness in general and the evaluated impact of psoriasis. The survey was pre-tested by three psoriasis patients and, based on the resulting feedback, slightly modified. For the scales used in this study, internal consistency was considered "excellent" for Cronbach's alpha values $>0.9$ and "good" for Cronbach's alpha values > 0.8 [36].

\section{Subjective well-being}

Following Diener et al. [25], subjective well-being was operationalized as $P A, N A$ and $S W L$. PA and NA were measured using the validated German version of the Scale of Positive and Negative Experience (SPANE) [37, 38]. We chose the SPANE over the more frequently used Positive and Negative Affect Schedule (PANAS) [39], as it assesses a wider range of positive and negative emotions than the PANAS, which focusses on high arousal emotions, and as it is coherent with Diener and colleagues conceptualization of subjective well-being [37]. The SPANE consists of two subscales measuring PA and NA, respectively. Each subscale consists of six adjectives, e.g., "pleasant" and "positive" for PA and "unpleasant" and "negative" for NA). The respondents were asked to indicate how often they had felt the respective feelings over the past two weeks on a 5-point scale from 1 ("very rarely or never") to 5 ("very often or always"). The two distinct subscales, showed excellent and good reliability in this study with Cronbach's alphas of 0.93 and 0.86 for PA and NA, respectively. For each subscale, the items were averaged to form an index. $S W L$ was measured using the German version of the Satisfaction With Life Scale (SWLS) [40, 41]. The scale consists of five items (e.g., "in most ways my life is close to my ideal"), each rated on a 7-point scale from 1 ("strongly disagree") to 7 ("strongly agree"). The scale showed excellent reliability in this study with a Cronbach's alpha of 0.91 and the items were averaged to form an index. As some researchers argue that happiness is not in fact a multidimensional but a unidimensional construct [29], we decided to also include a heuristic measure of happiness, meaning a single question asking participants for their overall happiness [20]. In order to achieve comparability with the general population, a single question from the European Social Survey was used: "Taking all things together, how happy would you say you are?" [42]. Respondents could give their answer on a 11-point scale from 0 ("extremely unhappy") to 10 ("extremely happy").

As an additional question on happiness in the context of psoriasis, the participants were asked for a subjective evaluation of the impact of psoriasis on their own happiness ("Do you think that your psoriasis has a negative impact on how happy you are?"-“No", "Yes, a little", "Yes, moderately", "Yes, very much").

\section{Depression}

The WHO-5 Well-Being Index [WHO-5, 43] is a validated screening questionnaire for depression. It consists of five statements (e.g., "My daily life has been filled with things that interest me"), which respondents rate on a 6-point scale from 0 ("at no time") to 5 ("all of the time"). The items showed good reliability in this study with a Cronbach's alpha of 0.87 . Following the instructions, the values of the items were added, resulting in an overall score ranging from 0 to 25 , with lower scores indicating a higher risk for depression. Using a cut-off of $\leq 7$, the WHO-5 has shown good properties for the screening of major depression with a sensitivity of $94 \%$ and specificity of $78 \%$ [44]. Consequently, a score of $\leq 7$ was considered a positive screening result for depression in this study.

\section{Participants' characteristics}

As possible parameters associated with happiness, data on age (in years), gender, years since first diagnosis, and current treatment status (currently receiving treatment vs. not receiving treatment) were collected. Subjective general disease severity was measured as "mild", "moderate", "severe". In addition, data on current disease severity were collected using the same given options ("mild", "moderate", "severe"). Based on general and current disease severity, new dichotomous variables indicating current phases of relative improvement or relative deterioration compared to general disease severity were derived.

\section{Analysis}

Prior to the analysis, data were checked for completeness (at least $80 \%$ of questions on happiness answered) and plausibility (e.g., data were considered implausible if the participants indicated a higher number of years since first diagnosis than age). As only very few participants did not meet our criteria 
for completeness and plausibility $(n=8)$, these cases were excluded from all further analysis rather than using imputation in order to keep the analysis as simple as possible. The remaining data were analyzed descriptively. Pearson's correlations were calculated in order to explore associations between the examined variables. Correlations were considered moderate for $r>0.5$ and strong for $r>0.7$ [45]. To further differentiate the four constructs PA, NA, SWL, and depression, exploratory factor analysis using Promin Rotation, an oblique rotation method appropriate for correlated variables, was conducted after checking the respective requirements were fulfilled. As all variables were measured on at least 5-step scales (with equal distances between the answer options and numbers suggesting equal steps between the categories), variables were treated as quasi-metric and Pearson's correlations were used for factor analysis. Following Guadagnoli and Velicer [46], factor loadings of 0.4 and higher were considered stable, which is why all smaller coefficients were suppressed. Factor retention was determined using parallel analysis [47]. However, as the retention of three factors, which was the number of factors suggested by parallel analysis, did not result in stable factor loadings for all examined items, we additionally conducted the analysis retaining one more factor, which was in line with the theoretical assumption of four differing constructs of PA, NA, SWL, and depression.

Means of PA, NA, heuristic happiness, SWL and WHO-5 were compared to norm data [41, 48, 49] or, in case of PA and NA, to data of a validation study [38] as norm data were not available. For both SWL and WHO-5, norm data had been collected in representative samples of the German general population (with the assistance of a demographic consulting company (SWL: mean age $48.9 \pm 18.3$ years, $52.2 \%$ women; WHO-5: mean age 48.3 years, SD not indicated for the overall sample, $52.7 \%$ women) [41, 48]. For heuristic happiness, norm data for the German general population were collected within the European Social Survey (mean age $48.2 \pm 18.1$ years, $49.9 \%$ women) [49]. For PA and NA, the data of the German validation study among a total of 498 participants recruited in university lectures $(n=264)$ and via mailing lists/social media $(n=234)$ were used as reference data (mean age not indicated, $18.5 \%<20$ years, $47.8 \%$ 20-29 years, $16.3 \% 30-39$ years, $7.7 \%$ 40-49 years, $5.3 \%$ $50-59$ years, $4.5 \%>59$ years, $75.1 \%$ women) [38]. As for PA, NA, and heuristic happiness the datasets of the reference samples were accessible, the group comparisons for these variables were conducted using ANCOVA and planned contrasts while controlling for sex and age. Adjusted means $\left(\mathrm{m}^{\mathrm{a}}\right)$ are reported. For SWL and WHO5 , the datasets of the reference populations could not be retrieved, which is why analyses were conducted stratified for age and sex, using Student's $t$-tests. The age groups for these analyses were chosen to match the age groups reported in the respective reference samples. As Student's $t$-tests and ANCOVA have been shown to provide robust results even when normality and equal variance assumptions are violated [50-52], the analyses were conducted without prior verification of these assumptions.

Parameters associated with subjective well-being and a positive screening for depression were identified using multiple linear regression models and binary logistic regression, respectively. In all regression models, age, gender, years since first diagnosis, general subjective disease severity, improvement or deterioration of skin condition and current treatment status were entered as independent variables. As a result, adjusted raw (B) and standardized regression coefficients $(\beta)$ and Odds Ratios (OR) as well as corresponding 95\%-confidence intervals (CIs) and the percentage of variance explained by each model (adjusted $\mathrm{R}^{2}$ ) are reported. As depression is characterized by the inability to feel happy [35], we conducted additional sub-group analyses in participants who did not receive a positive screening result for depression in order to explore differential findings for affective well-being which are not explained by the presence of depression.

The level of significance was set at $\alpha=0.05$ for all analyses. All statistical analyses except exploratory factor analysis were conducted using IBM SPSS Statistics Version 24 (IBM Corporation, Armonk, NY, USA). Exploratory factor analysis was conducted using FACTOR software [53].

\section{Results}

In total, 730 participants completed the survey, with most users having participated via "Psoriasis Netz" (95\%). Of those, eight were excluded prior to analysis due to insufficient $(n=3)$ or implausible data (e.g., higher number of years since first diagnosis than age; $n=5$ ). Consequently, the data of 722 participants were analyzed. The mean age was 45.8 years with a standard deviation (SD) of 13.4 years and a range of $12-85$ years (Table 1). $62.7 \%$ of participants were women. Almost all participants $(98.6 \%)$ reported having been diagnosed with psoriasis by a medical doctor, and only ten participants (1.4\%) had been given the diagnosis by an alternative practitioner or had self-diagnosed psoriasis. On average, the participants had been living with psoriasis for 20.6 years $(\mathrm{SD}=14.5$ years, range $<1-68)$. The majority of participants $(55.7 \%)$ judged their psoriasis to be generally moderate, $34.3 \%$ severe, and only $10 \%$ mild. When asking for current disease severity, $24 \%$ of participants indicated mild, $52.8 \%$ moderate, and $23.3 \%$ severe. Comparing selfreported general and current disease severity, $31.7 \%$ of participants were in a phase of relative improvement, and $14.4 \%$ 
Table 1 Baseline patients' characteristics

\begin{tabular}{ll}
\hline Patients' characteristics & $N=722$ \\
\hline Age in years (mean \pm SD, range) & $48.8 \pm 13.4,12-85$ \\
Gender & \\
- Male & $269(37.3 \%)$ \\
- Female & $453(62.7 \%)$ \\
Diagnosis & \\
- By medical doctor & $712(98.6 \%)$ \\
- By alternative practitioner or self-diagnosis & $10(1.4 \%)$ \\
Years since first diagnosis (mean \pm SD, range) & $20.6 \pm 14.5,0-68$ \\
General disease severity & \\
- Mild & $72(10 \%)$ \\
- Moderate & $402(55.7 \%)$ \\
- Severe & $248(23.3 \%)$ \\
Current disease severity & \\
- Mild & $173(24 \%)$ \\
- Moderate & $381(52.8 \%)$ \\
- Severe & $168(23.3 \%)$ \\
Phase of relative improvement of skin condition & $229(31.7 \%)$ \\
Phase of relative deterioration of skin condition & $104(14.4 \%)$ \\
Current treatment ${ }^{\mathrm{a}}$ & \\
- Dermatologist & $446(61.8 \%)$ \\
- Rheumatologist & $127(17.6 \%)$ \\
- General practitioner & $117(16.2 \%)$ \\
- Alternative care & $23(3.2 \%)$ \\
- No current treatment & $179(24.8 \%)$ \\
\hline
\end{tabular}

${ }^{\text {a }}$ Multiple answers were possible

of relative deterioration. One out of four participants (24.8\%) was not currently receiving medical treatment for psoriasis. Of those who were receiving treatment, the majority $(82.1 \%)$ were treated by a dermatologist, $23.3 \%$ by a rheumatologist, $21.5 \%$ by a general physician, and $3.2 \%$ received alternative care (e.g., by an alternative practitioner).

\section{Subjective well-being and depression}

The variables intercorrelated moderately to strongly, with the strongest correlation observed for PA and heuristic happiness $(r=0.73, p<0.001$; Table 2$)$ and the weakest correlation observed for SWL and NA $(r=-0.56, p<0.001)$. $40.3 \%$ of participants had a positive screening result for depression ( $41.9 \%$ of women and $37.5 \%$ of men). Nine out of ten participants $(90.3 \%)$ stated that psoriasis had a negative impact on how happy they were (28.8\% a little, $29 \%$ moderately, and $32.9 \%$ very much), and only $9.3 \%$ of participants stated that their psoriasis did not affect their happiness at all (Fig. 1).

Using exploratory factor analysis and parallel analysis for factor retention, three factors were identified (Fig. 2a). The first factor corresponded to the PA items of the SPANE, the second corresponded to the items of the SWLS, and the third factor corresponded to the NA items of the SPANE, with one item of the WHO-5 loading on the same factor. The remaining items of the WHO-5 did not show stable factor loadings on any of the three factors. When adding one more factor to be extracted, all items of SPANE, SWLS, and WHO-5 loaded on four separate factors corresponding to the four constructs PA, NA, SWL and depression (Fig. 2b).

Descriptive statistics of PA, NA, SWL, heuristic happiness, and WHO-5 are displayed in Table 3.

\section{Comparison with reference populations}

Controlling for age and sex, participants in this study reported significantly less PA than the German validation sample ( $m^{\mathrm{a}}=2.98$ vs. 3.10, $p=0.003$ ) [38], but did not differ from them in terms of NA ( $m^{\mathrm{a}}=2.87$ vs. $2.88, p=0.767$; Table 3). Furthermore, controlling for age and sex, participants in this study judged themselves less happy than the general population $\left(m^{\mathrm{a}}=6.34\right.$ vs. $\left.7.26, p<0.0001\right)$. Stratifying for age and sex, participants of this study over all age groups and both men and women scored significantly lower on the WHO-5 than the general population (all $p<0.001$ ).

Table 2 Correlation matrix

\begin{tabular}{llllll}
\hline & PA & NA & SWL & Heuristic happiness & $\begin{array}{l}\text { WHO-5 } \\
\text { well-being } \\
\text { index }\end{array}$ \\
\hline PA & 1 & & & \\
NA & $-0.669^{* * *}$ & 1 & & & \\
SWL & $0.652^{* * *}$ & $-0.564 * * *$ & 1 & & 1 \\
Heuristic happiness & $0.728^{* * * *}$ & $-0.588^{* * *}$ & $0.629 * * *$ & 1 & \\
WHO-5 well-being index & $0.687^{* * *}$ & $-0.651^{* * *}$ & $0.630^{* * *}$ & $0.591 * * *$ & \\
\hline
\end{tabular}

Pearson's correlation coefficients are displayed

Asterisks indicate significant correlations; ${ }^{*} p<0.05,{ }^{* *} p<0.01,{ }^{* * *} p<0.001$ 


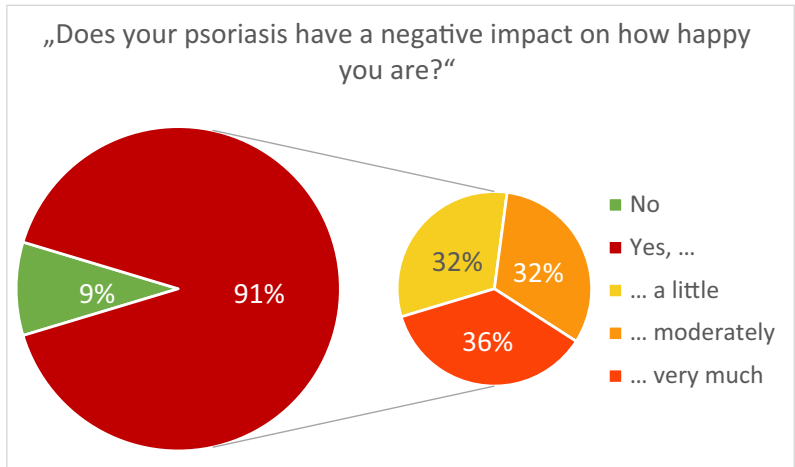

Fig. 1 Subjective evaluation of the impact of psoriasis on patients' happiness. $N=721$, one missing case

Furthermore, stratified analyses showed that participants under 65 years in this sample were less satisfied with their lives than the general population under 65 years (all $p<0.001$ ), while participants of 65 years and older did not differ from their peers in terms of SWL $(p>0.05)$. Taking all age groups together, both men and women in this study reported lower SWL than men and women of the general population (both $p<0.0001$ ).

\section{(a) Factor retention based on parallel analysis}

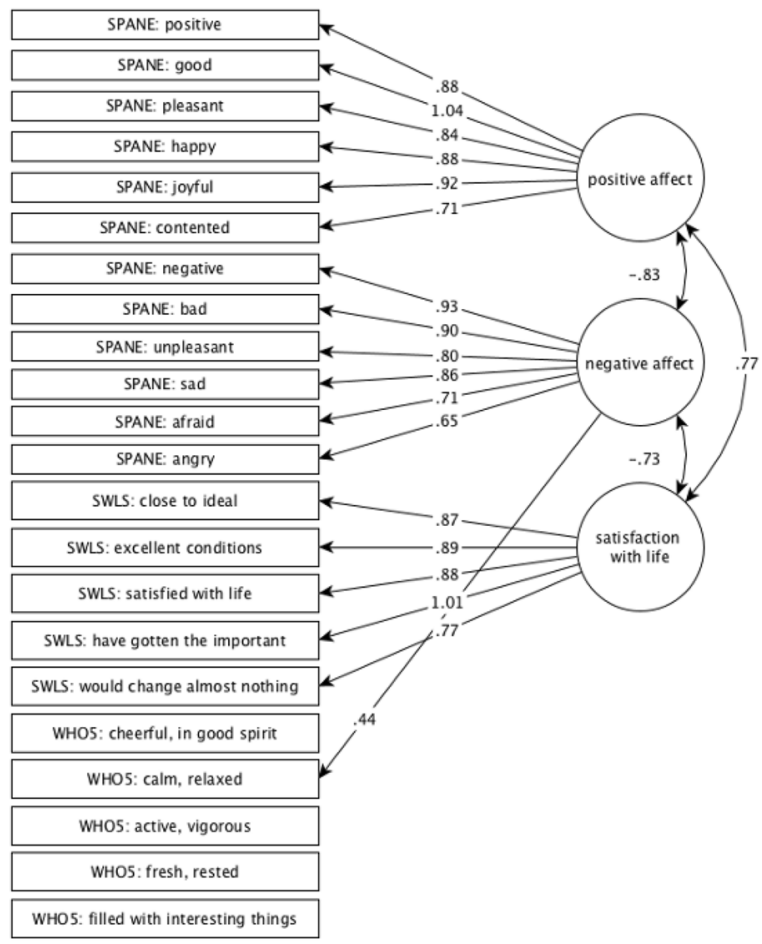

Fig. 2 Factor loadings of the examined variables of the SPANE, the SWLS, and the WHO-5 a on the three factors retained based on parallel analysis $\mathbf{b}$ on four factors corresponding to the four constructs

\section{Factors associated with subjective well-being and depression}

\section{$\mathrm{PA}$}

Higher age $[\mathrm{B}=-0.01, \mathrm{CI}(-0.12 ;-0.002) ; \beta=-0.10, \mathrm{CI}$ $(-0.18 ;-0.03)]$, moderate $[\mathrm{B}=-0.33, \mathrm{CI}(-0.55 ;-0.11)$; $\beta=-0.18, \mathrm{CI}(-0.31 ;-0.06)]$ and severe $[\mathrm{B}=-0.85, \mathrm{CI}$ $(-1.11 ;-0.59) ; \beta=-0.45$, CI $(-0.59 ;-0.31)]$ general disease severity as compared to mild disease severity and deterioration of skin condition $[\mathrm{B}=-0.32$, $\mathrm{CI}(-0.53$; $-0.13) ; \beta=-0.13$, CI $(-0.2 ;-0.05)]$ were associated with lower levels of PA (Fig. 3). In contrast, a higher number of years since the first diagnosis $[\mathrm{B}=0.11, \mathrm{CI}(0.01 ; 0.02)$; $\beta=0.18, \mathrm{CI}(0.10 ; 0.25)]$ and improvement of skin condition $[\mathrm{B}=0.36$, CI $(0.21 ; 0.52) ; \beta=0.06$, CI $(0.11 ; 0.27)]$ were associated with higher PA. The full model explained $10.7 \%$ of variance in PA $(p<0.001)$. When including only patients without depression, the same parameters were associated with PA, but the model explained only $7.5 \%$ of variance $(p<0.001)$.

\section{(b) Factor retention based on theoretical framework}

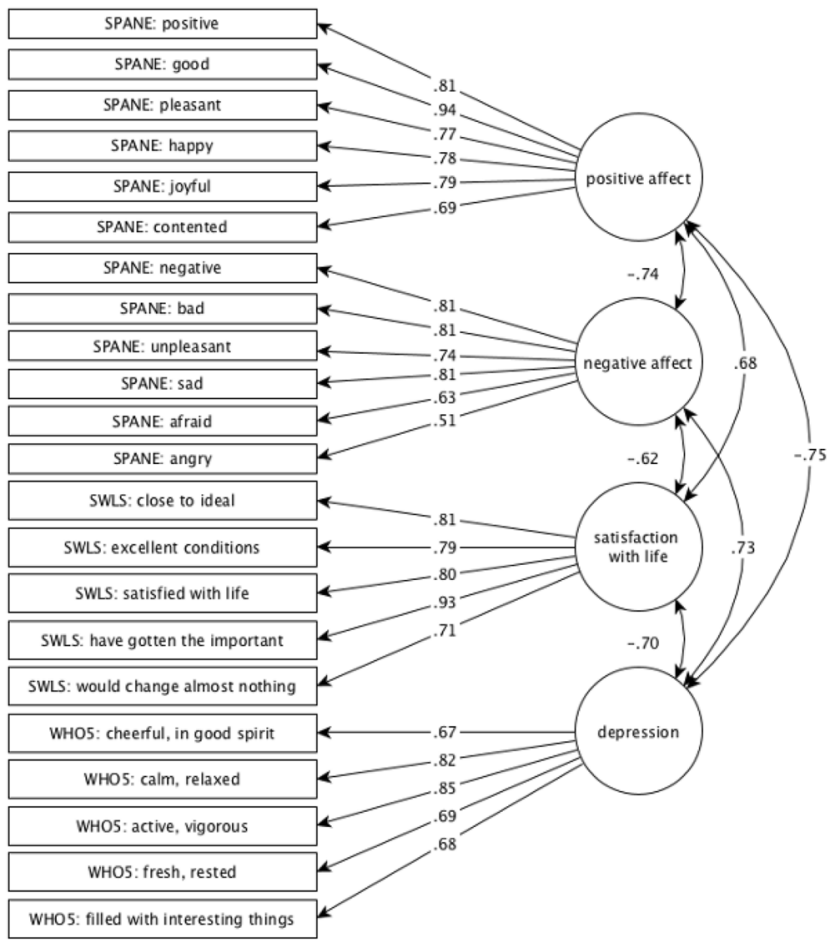

PA, NA, SWL, and depression. Arrows between factors show interfactor correlations 
Table 3 Descriptive statistics of the examined variables in the study sample and the respective reference samples

\begin{tabular}{|c|c|c|c|c|}
\hline & Mean \pm SD & $\begin{array}{l}\text { Adjusted mean/ } \\
\text { means per } \\
\text { group } \pm \mathrm{SD}\end{array}$ & $\begin{array}{l}\text { Adjusted mean/means per } \\
\text { group } \pm \mathrm{SD} \text { (reference) }\end{array}$ & Significance $^{\mathrm{a}}$ \\
\hline PA & $2.96 \pm 0.89$ & 2.98 & 3.10 & 0.003 \\
\hline NA & $2.83 \pm 0.89$ & 2.87 & 2.88 & 0.767 \\
\hline Heuristic happiness & $5.32 \pm 2.37$ & 6.34 & 7.26 & $<0.001$ \\
\hline SWL & $4.27 \pm 1.43$ & & & \\
\hline - 14-24 years & & $19.63^{\mathrm{b}} \pm 6.24$ & $24.77^{c} \pm 6.11$ & $<0.001$ \\
\hline - 15-34 years & & $21.87^{\mathrm{b}} \pm 7.47$ & $25.89^{c} \pm 6.34$ & $<0.001$ \\
\hline - 35-44 years & & $20.77^{\mathrm{b}} \pm 7.66$ & $25.01^{\mathrm{c}} \pm 6.37$ & $<0.001$ \\
\hline$-45-54$ years & & $20.80^{b} \pm 6.79$ & $24.43^{c} \pm 6.81$ & $<0.001$ \\
\hline - 55-64 years & & $21.28^{\mathrm{b}} \pm 7.28$ & $24.15^{\mathrm{c}} \pm 6.32$ & $<0.001$ \\
\hline - 65-74 years & & $23.73^{\mathrm{b}} \pm 6.13$ & $25.30^{c} \pm 5.52$ & 0.059 \\
\hline - > 74 years & & $24.56^{\mathrm{b}} \pm 3.78$ & $25.06^{\mathrm{c}} \pm 5.93$ & 0.802 \\
\hline - Men & & $20.86^{\mathrm{b}} \pm 7.40$ & $25.12 \pm 6.32$ & $<0.001$ \\
\hline - Women & & $21.62^{\mathrm{b}} \pm 7.05$ & $24.67 \pm 6.20$ & $<0.001$ \\
\hline WHO-5 well-being index & $9.86 \pm 5.27$ & & & \\
\hline$-<41$ years & & $9.73 \pm 4.96$ & $18.36 \pm 4.80$ & $<0.001$ \\
\hline - 41-60 years & & $9.41 \pm 5.30$ & $17.49 \pm 4.88$ & $<0.001$ \\
\hline - $>60$ years & & $11.9 \pm 5.65$ & $16.70 \pm 5.13$ & $<0.001$ \\
\hline - Men & & $10.34 \pm 5.61$ & $18.15 \pm 4.90$ & $<0.001$ \\
\hline - Women & & $9.58 \pm 5.05$ & $17.07 \pm 4.98$ & $<0.001$ \\
\hline
\end{tabular}

${ }^{a}$ ANCOVA and Student's $t$-tests, respectively

${ }^{b}$ For stratified analysis items were summed up (instead of averaged) in order to allow for comparison with reference data

${ }^{\mathrm{c} C a l c u l a t e d}$ based on weighted means for men and women
NA

NA was higher in participants with moderate $[\mathrm{B}=0.33$, CI $(0.11 ; 0.55) ; \beta=0.19, \mathrm{CI}(0.06 ; 0.31)]$ and severe $[\mathrm{B}=0.74, \mathrm{CI}(0.48 ; 1) ; \beta=0.40, \mathrm{CI}(0.26 ; 0.54)]$ general disease severity and those who were currently in a phase of deterioration of the skin condition $[\mathrm{B}=0.34$, $\mathrm{CI}(0.15$; $0.54) ; \beta=0.14$, CI $(0.06 ; 0.21) ;$ Fig. 3]. In contrast, being male $[\mathrm{B}=-0.16$, CI $(-0.29 ;-0.03) ; \beta=-0.09$, CI $(-0.16 ;-0.02)]$ and living with psoriasis for a longer time $[\mathrm{B}=-0.01$, CI $(-0.02 ;-0.006) ; \beta=-0.17$, CI $(-0.24 ;-0.09)]$ were associated with less NA. The full model explained $9.1 \%$ of variance in NA $(p<0.001)$. When including only patients without depression, the association remained significant for moderate and severe general disease severity and years since the diagnosis of psoriasis $(p=0.008 ; p=0.001 ; p<0.001$, respectively $)$, but the associations with gender and deterioration of the skin did not reach significance anymore $(p=0.071$ and $p=0.085$, respectively). Similar to PA, the model explained slightly less variance $(7.6 \%, p<0.001)$ in participants without depression compared to the whole sample.

\section{SWL}

Severe general disease severity was associated with lower levels of SWL $[\mathrm{B}=-1.04$, CI $(-1.47 ;-0.61) ; \beta=-0.34$, CI $(-0.49 ;-0.20)$; Fig. 3]. More years passed since the first diagnosis $[\mathrm{B}=0.01, \mathrm{CI} 0.002 ; 0.02) ; \beta=0.10, \mathrm{CI}(0.02$; $0.18)]$ and current improvement of skin condition $[\mathrm{B}=0.27$, CI $(0.01 ; 0.52) ; \beta=0.09, \mathrm{CI}(0.00 ; 0.17)]$ were associated with higher SWL. The full model explained $5.5 \%$ of variance $(p<0.001)$ in SWL. When excluding participants with depression, only female gender was significantly associated with SWL $(p=0.049)$. Overall, the model did not show a good fit for this sub-sample (adjusted $\mathrm{R}^{2}=0.013, p=0.099$ ).

\section{Heuristic happiness}

Patients who were in general moderately $[\mathrm{B}=-1.11$, CI $(-0.1 .7 ;-0.51) ; \beta=-0.24$, CI $(-0.36 ;-0.11)]$ or severely $[\mathrm{B}=-2.28$; CI $(-2.97 ;-1.59) ; \beta=-0.46$, CI $(-0.60 ;-0.32)]$ affected or who were currently in a phase of deterioration $[\mathrm{B}=1.1, \mathrm{CI}(0.69 ; 1.51) ; \beta=-0.14$, CI $(-0.22 ;-0.07)]$ judged themselves to be less happy 


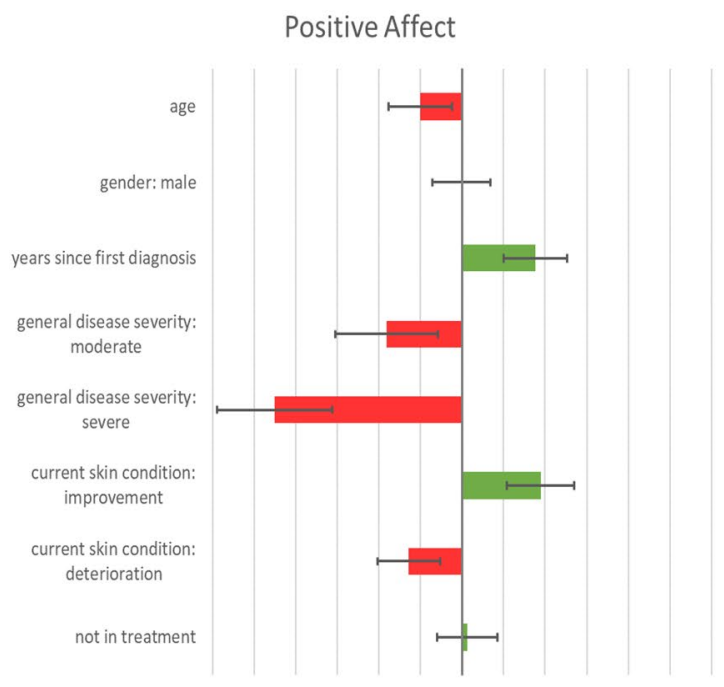

$\begin{array}{lllllllllllll}-0,6 & -0,5 & -0,4 & -0,3 & -0,2 & -0,1 & 0 & 0,1 & 0,2 & 0,3 & 0,4 & 0,5 & 0,6\end{array}$

Satisfaction With Life

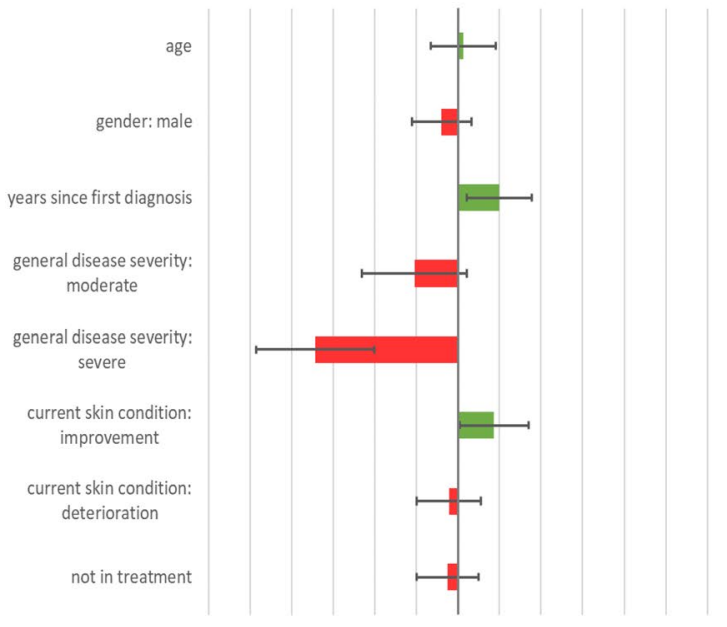

$\begin{array}{lllllllllllll}-0,6 & -0,5 & -0,4 & -0,3 & -0,2 & -0,1 & 0 & 0,1 & 0,2 & 0,3 & 0,4 & 0,5 & 0,6\end{array}$

WHO-5

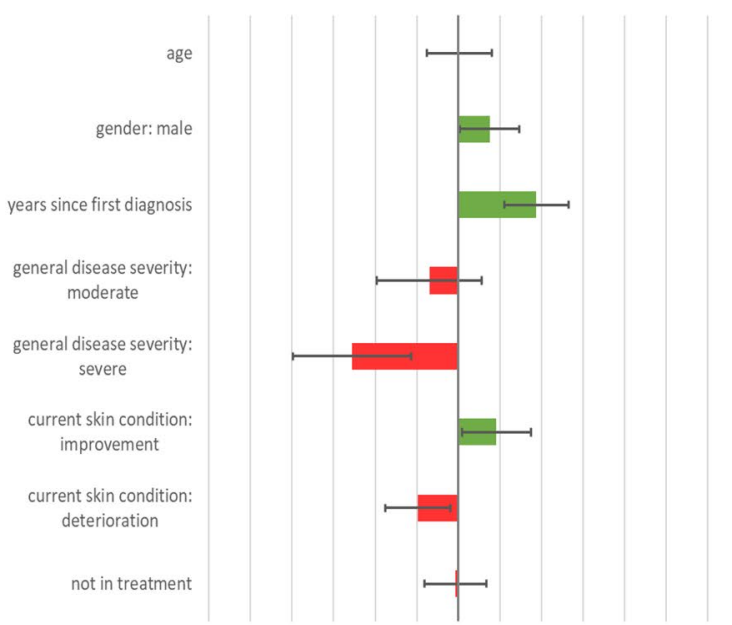

$\begin{array}{lllllllllllll}-0,6 & -0,5 & -0,4 & -0,3 & -0,2 & -0,1 & 0 & 0,1 & 0,2 & 0,3 & 0,4 & 0,5 & 0,6\end{array}$
Negative Affect

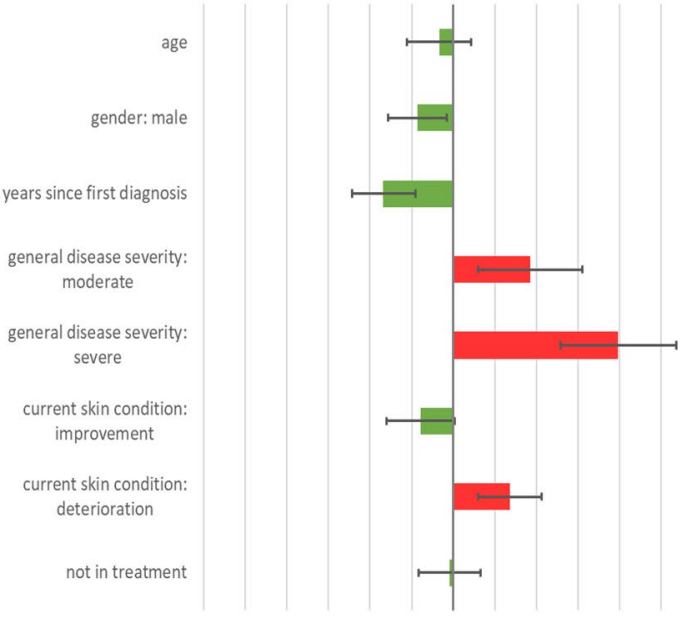

$\begin{array}{lllllllllllll}-0,6 & -0,5 & -0,4 & -0,3 & -0,2 & -0,1 & 0 & 0,1 & 0,2 & 0,3 & 0,4 & 0,5 & 0,6\end{array}$

Heuristic Happiness

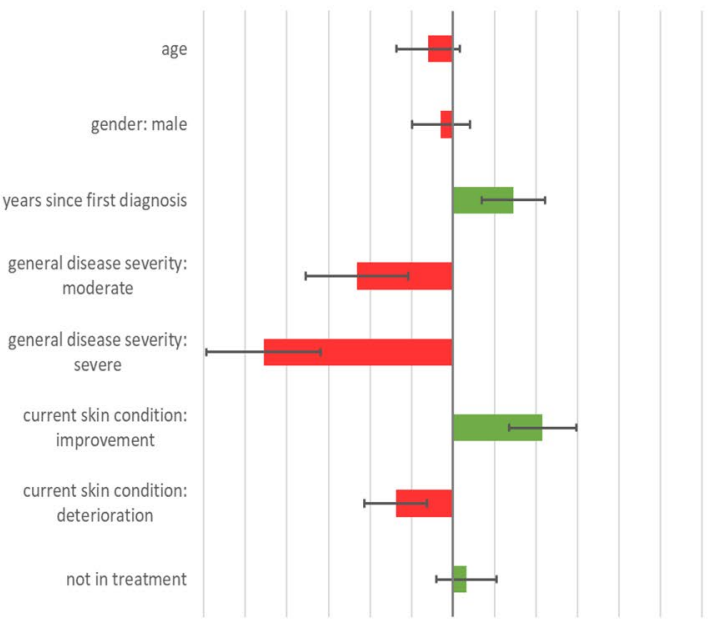

$\begin{array}{lllllllllllll}-0,6 & -0,5 & -0,4 & -0,3 & -0,2 & -0,1 & 0 & 0,1 & 0,2 & 0,3 & 0,4 & 0,5 & 0,6\end{array}$

Positive Screening for Depression

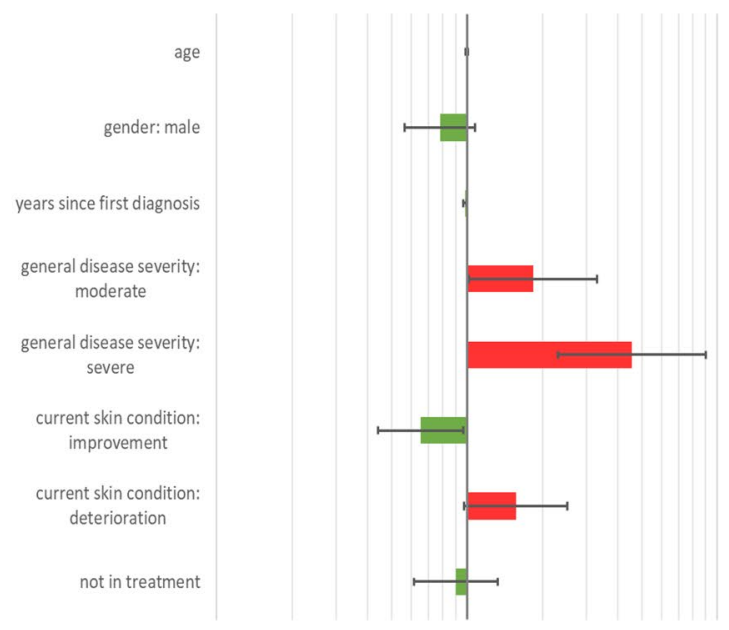


4Fig. 3 Determinants of happiness and depression in 722 individuals with psoriasis. Adjusted standardized regression coefficients (for positive screening of depression: OR on $\log$ scale) with corresponding confidence intervals are given. Factors associated with higher wellbeing are displayed in green and those associated with lower wellbeing are displayed in red. Happiness was operationalized as subjective well-being, consisting of PA, NA, and SWL, and as a heuristic evaluation of happiness. A screening result for depression was considered positive if a participant scored $\leq 7$ in the WHO-5. Asterisks indicate significant correlations. ${ }^{*} p<0.05,{ }^{*} p<0.01, * * * p<0.001$. (Color figure online)

(Fig. 3). In contrast, those who had been living with psoriasis for more years $[\mathrm{B}=0.02, \mathrm{CI}(0.01 ; 0.04] ; \beta=0.14$, CI $(0.07 ; 0.22)]$ or who were in a current phase of improvement of skin condition $[\mathrm{B}=1.1, \mathrm{CI}(0.69 ; 1.51) ; \beta=0.22$, CI $(0.14 ; 0.30)]$ judged themselves to be happier. The full model explained $10.2 \%$ of variance in heuristic happiness. All parameters associated with heuristic happiness remained significant when excluding patients with depression, and the model explained $6.9 \%$ of variance in heuristic happiness $(p<0.001)$.

\section{Depression}

Participants with severe psoriasis $[\mathrm{B}=-2.84$, CI $(-4.41$; $-1.27) ; \beta=-0.26$, CI $(-0.40 ;-0.11)]$ and currently going through a phase of deterioration $[\mathrm{B}=-1.46$, CI $(-2.62$; $-0.3) ; \beta=-0.10$, CI $(-0.18 ;-0.02)]$ scored lower in the WHO-5 questionnaire, which indicates higher levels of depressive tendencies (Fig. 3). In contrast, men $[\mathrm{B}=0.82$, $\mathrm{CI}(0.04 ; 1.6) ; \beta=0.08, \mathrm{CI}(0.01 ; 0.15)]$, participants living with psoriasis for a longer time $[\mathrm{B}=0.07, \mathrm{CI}(0.04 ; 0.1)$; $\beta=0.19, \mathrm{CI}(0.11 ; 0.27)]$ and those currently experiencing improvement of skin condition $[\mathrm{B}=1.04$, CI $(0.11 ; 1.98)$; $\beta=0.09$, CI $(0.01 ; 0.18)]$ scored higher in the WHO-5. The full model explained $6.6 \%$ of variance in the WHO-5 score $(p<0.001)$. Moderately affected participants had a 1.8-fold [CI $(1.02 ; 3.32)]$ and severely affected participants even had a 4.6 -fold [CI $(2.31 ; 9.03)]$ chance of being screened positive for depression compared to mildly affected participants (Fig. 3). Living with psoriasis for a year longer [OR 0.98, CI $(0.97 ; 0.99)]$ and current improvement of skin condition [OR 0.65 , CI $(0.44 ; 0.97)]$ were both associated with a decreased chance of a positive screening for depression. The full model explained $8.6 \%$ of variance in screening results $(p<0.001)$.

\section{Discussion}

This study examined both subjective well-being and depression in patients with psoriasis, following the WHO's demand for a holistic approach of measuring mental health in this patient group [12]. In a nationwide sample of 722 individuals affected by psoriasis in Germany, subjective well-being and more precisely PA and SWL were low compared to the general population or healthy reference populations, and $40 \%$ of participants were screened positive for depression. The main risk factor for low subjective well-being and depression was general disease severity, but also deterioration of the skin was negatively associated with the affective component of subjective well-being, while phases of improvement of skin condition were associated with more PA and higher SWL. Additionally, the longer participants had been living with psoriasis, the higher was their subjective well-being and the less depressed they were.

\section{Differentiating depression and well-being}

Due to the simultaneous assessment of depression and subjective well-being, this study allowed to examine the association between these constructs in individuals affected with psoriasis. As depression, among other symptoms, is characterized by the absence of PA [35], the constructs of depression and subjective well-being/positive affect are bound to overlap per definition, which is reflected by overlapping items of the respective measurement tools (especially question 1 of the WHO- 5 and the PA items of the SPANE) [54]. However, depression is also characterized by other symptoms such as lack of energy and decreased activity which are not covered by PA/subjective well-being. Accordingly, exploratory factor analysis revealed that all items of SPANE, SWLS and WHO-5 loaded on four different factors corresponding to PA, NA, SWL and depression, supporting the methodological approach of differentiating depression and subjective well-being. This is especially interesting as the empirical differentiation of subjective well-being and depression, which has been successfully shown in some studies [55], has proven difficult in others [56]. The results of this study are in line with the postulate of the WHO that well-being is more than the absence of illbeing (see also Lukat et al. [57]). Thereby, they underline the WHO's demand to including measures of well-being in clinical research and practice in individuals with psoriasis in order to achieve a more comprehensive understanding of the mental burden of this disease [12].

\section{Subjective and objective disease severity}

Subjective general disease severity was associated with all facets of subjective well-being in this study. In contrast, two previous studies have only found associations between disease severity and single facets of subjective well-being (NA: Martin-Brufau et al. [23]; PA: Schuster et al. [20], respectively). These inconsistencies in the findings might be owed to the different modalities of assessing disease severity employed in the different studies: Previous studies 
have shown that the subjective perception of the symptoms might be more important for individual well-being than the objective course of the disease [21, 26, 27]. While the two mentioned studies used either physician assessed disease severity [20] or a validated self-assessment tool for disease severity [23], a very subjective approach was used in the present study, with participants rating their disease severity as "mild", "moderate" or "severe", This approach might explain why the associations between well-being and disease severity were especially pronounced in this study. More research is needed in order to shed light on the (different?) roles of subjective and objective disease severity for wellbeing and mental health. An alternative explanation could be that the small sample size of 52 patients in the study of Schuster et al. [20] prevented the detection of associations between disease severity and NA and SWL observed in this study.

\section{General and current disease severity}

Interestingly, high general disease severity remained strongly associated with low subjective well-being and depression even when current skin condition was taken into account. While improvement of the skin was associated with higher well-being, the negative association between general severe disease severity and well-being was more pronounced. These findings implicate that by relieving skin symptoms, physicians might be able to help patients feel happier and, as reported before $[15,16]$, reduce the risk for depression. However, especially in more severe forms of psoriasis, wellbeing might remain low even if the symptoms improve. This supports the idea that documenting the PeakPASI, meaning the highest PASI ever recorded per patient, might provide additional information about the mental burden of psoriasis compared to just referring to the "snapshot" PASI at a single visit [58].

\section{The role of online recruitment}

The participants in this sample scored lower than the German norm in SWL and reported less PA than a healthy reference sample. While the findings of impaired PA, but not increased NA, is in line with previous findings [20], the reported values for PA, NA and SWL were even more unfavorable than in a previous study among patients with psoriasis [20]. Also, we found a high rate of positive screening for depression of $40 \%$. An explanation for these findings could be that the recruitment strategy might have led to a sample which was especially impaired in terms of well-being and depression. This would suggest that especially unhappy individuals turn to (online offers of) self-help organizations like the one used for recruitment in this study, which would underline the great importance these entities have for the comprehensive treatment of individuals with psoriasis. In contrast, however, it is also possible that, due to the anonymity, the participants in this online survey were more honest and open about their well-being than in previous studies, which have mostly taken place in medical settings [20, 24, 32]. Thus, social desirability might have led to an underestimation of happiness and well-being in previous studies.

\section{Limitations}

The results of this study are subject to several limitations. First, as the survey was conducted online, selection bias must be considered, which was already discussed in the above paragraph. Second, while all participants had to actively indicate that they were indeed affected by psoriasis prior to taking part in the survey, the online setting did not allow to confirm the diagnosis. Third, due to time limitations in the survey, which was designed to take only a few minutes to complete in order to achieve a high number of participants, disease severity was not assessed using validated scales or clinical scores but as "mild", "moderate" and "severe". Future studies should explore whether the findings of this study can be replicated when using validated scores for the assessment of subjective or even objective disease severity like PASI. Also, as this is a cross-sectional study, we could not determine causality but only association. Finally, future studies should explore the role of personality traits and other possible moderator variables such as marital status for the examined associations, as they have not been considered in this study.

\section{Strengths}

This study followed a holistic approach of assessing mental health in individuals with psoriasis by measuring not only depression, but also subjective well-being in a large sample of affected individuals. Thus, the study does not only contribute to the growing body of research exploring wellbeing in psoriasis healthcare, it furthermore adds empirical evidence supporting the differentiation of depression and well-being as two related constructs. Furthermore, the study was designed in a way to not only include patients in the medical setting, but also affected individuals who were not currently part of the health care system, which is in line with the WHO's demand for "people centered care" instead of "patient centered care" [59]. Thus, by looking at people with psoriasis in general instead of focusing on patients only (= people who are currently receiving medical care), the study provides a more comprehensive picture of mental health in individuals with psoriasis regardless of treatment status than prior studies which were mostly conducted in medical settings. Finally, the online setting of this study allowed the participants to fill in the questionnaire in an 
anonymous setting, which might help to reduce social desirability bias as compared to paper-based questionnaires at doctors' offices or clinics which are frequently employed in epidemiological research.

\section{Future research}

Interventions targeting NA (especially stress) have been successfully conducted in patients with psoriasis $[60,61]$. As this and previous studies have shown that PA seems to be especially impaired in patients with psoriasis [20], and as PA has been linked to several desirable health outcomes like better immune reactions, faster skin barrier recovery, and better cardiovascular health [62, 63], future research should evaluate whether interventions targeting PA could be also or maybe even more beneficial for improving the patients' well-being beyond the treatment of skin symptoms. Furthermore, future research should explore the mechanisms behind the presumably beneficial effect of skin treatment on wellbeing, as it could be moderated and potentially improved by additional factors such as time to treatment response [26].

\section{Conclusion}

In this large sample of 722 patients with psoriasis, participants reported lower subjective well-being than the German general population or healthy reference populations. General disease severity was associated with both low subjective well-being and depression, even in phases of improvement of the skin condition. While improvement of skin condition, which can be achieved by an effective treatment of skin symptoms, was associated with higher well-being and less depression, the negative associations between severe general disease severity and well-being and depression, respectively, were even more pronounced. As subjective well-being and depression have been identified as two differential constructs in this study, measures of well-being should be increasingly incorporated in psoriasis health care.

\begin{abstract}
Acknowledgements We thank all participants for taking part in this study. We further thank Claudia Liebram and Rolf Blaga of "Psoriasis Netz" (www.psoriasis-netz.de) for the valuable support during the development of the study questionnaire and the set-up of the study and the recruitment of participants. Finally, we want to thank Dr. Bernd Neidl from "Farbenhaut" (www.farbenhaut.de) and the "Bitte berühren" team of the Professional Association of the German Dermatologists (BVDD) for sharing the link to the study questionnaire on their respective Facebook pages.
\end{abstract}

Author contributions BS, LT, SZ, MCS: Conceptualization; BS, CP, MCS, AZ: methodology; BS: software; LT, SZ, MCS, AZ: validation; BS: formal analysis; TB, AZ: resources; BS: writing—original draft preparation; CP, LT, SZ, MCS, AZ: writing-review and editing; TB,
AZ: supervision; MCS: project administration. All authors have read and agreed to the published version of the manuscript.

Funding Open Access funding enabled and organized by Projekt DEAL. The research was funded by the Department of Dermatology and Allergy, Technical University of Munich.

Data availability Data are available at http://dx.doi.org/10.23668/psych archives.4451.

Code availability Code is available upon request.

\section{Declarations}

Conflict of interest The authors declare that they have no conflict of interest.

Ethical approval This study was reviewed and approved by the local ethics committee of Technical University of Munich (reference 25/19 S).

Consent to participate All participants provided informed consent prior to participation.

Open Access This article is licensed under a Creative Commons Attribution 4.0 International License, which permits use, sharing, adaptation, distribution and reproduction in any medium or format, as long as you give appropriate credit to the original author(s) and the source, provide a link to the Creative Commons licence, and indicate if changes were made. The images or other third party material in this article are included in the article's Creative Commons licence, unless indicated otherwise in a credit line to the material. If material is not included in the article's Creative Commons licence and your intended use is not permitted by statutory regulation or exceeds the permitted use, you will need to obtain permission directly from the copyright holder. To view a copy of this licence, visit http://creativecommons.org/licenses/by/4.0/.

\section{References}

1. Michalek, I. M., Loring, B., \& John, S. M. (2017). A systematic review of worldwide epidemiology of psoriasis. Journal of the European Academy of Dermatology and Venereology, 31(2), 205-212. https://doi.org/10.1111/jdv.13854

2. Tizek, L., Schielein, M. C., Seifert, F., Biedermann, T., Bohner, A., \& Zink, A. (2019). Skin diseases are more common than we think: Screening results of an unreferred population at the Munich Oktoberfest. Journal of the European Academy of Dermatology and Venereology, 33(7), 1421-1428. https://doi.org/10.1111/jdv. 15494

3. Boehncke, W.-H., \& Schön, M. P. (2015). Psoriasis. The Lancet, 386(9997), 983-994. https://doi.org/10.1016/s0140-6736(14) 61909-7

4. Damiani, G., Cazzaniga, S., Conic, R. R., \& Naldi, L. (2019). Pruritus characteristics in a large Italian cohort of psoriatic patients. Journal of the European Academy of Dermatology and Venereology, 33(7), 1316-1324. https://doi.org/10.1111/jdv.15539

5. Pithadia, D. J., Reynolds, K. A., Lee, E. B., \& Wu, J. J. (2019). Psoriasis-associated cutaneous pain: Etiology, assessment, impact, and management. The Journal of Dermatological Treatment, 30(5), 435-440. https://doi.org/10.1080/09546634.2018.1528330 
6. Zink, A., Herrmann, M., Fischer, T., Lauffer, F., Garzorz-Stark, N., Bohner, A., et al. (2017). Addiction: An underestimated problem in psoriasis health care. Journal of the European Academy of Dermatology and Venereology, 31(8), 1308-1315. https://doi. org/10.1111/jdv.14204

7. Dalgard, F. J., Gieler, U., Tomas-Aragones, L., Lien, L., Poot, F., Jemec, G. B. E., et al. (2015). The psychological burden of skin diseases: A cross-sectional multicenter study among dermatological out-patients in 13 European countries. The Journal of Investigative Dermatology, 135(4), 984-991. https://doi.org/10. 1038/jid.2014.530

8. Tomas-Aragones, L., \& Marron, S. E. (2016). Body image and body dysmorphic concerns. Acta Dermato Venereologica, 96(217), 47-50. https://doi.org/10.2340/00015555-2368

9. Dimitrov, D., \& Szepietowski, J. C. (2017). Stigmatization in dermatology with a special focus on psoriatic patients. Postępy Higieny i Medycyny Doświadczalnej (Online), 71, 1115-1122. https://doi.org/10.5604/01.3001.0010.6879

10. Hrehorow, E., Salomon, J., Matusiak, L., Reich, A., \& Szepietowski, J. C. (2012). Patients with psoriasis feel stigmatized. Acta Dermato Venereologica, 92(1), 67-72. https://doi.org/10.2340/ 00015555-1193

11. Leisner, M. Z., Riis, J. L., Schwartz, S., Iversen, L., Ostergaard, S. D., \& Olsen, M. S. (2019). Psoriasis and risk of mental disorders in Denmark. JAMA Dermatology, 155(6), 745-747. https://doi. org/10.1001/jamadermatol.2019.0039

12. World Health Organization. (2016). Global report on psoriasis. Retrieved July 2, 2018, from http://www.who.int/iris/handle/ $10665 / 204417$

13. World Health Organization. (2014). Sixty-seventh world health assembly: Solutions and decisions. Retrieved August 3, 2020, from https://apps.who.int/gb/ebwha/pdf_files/WHA67-REC1/ A67_2014_REC1-en.pdf?ua $=1 \#$ page $=25$

14. Schielein, M. C., Tizek, L., Knobloch, L., Maßßen, D., Biedermann, T., \& Zink, A. (under review). Psoriasis and addiction: Assessing mental health with a focus on depression and anxiety in patients with psoriasis across Germany.

15. Strober, B., Gooderham, M., de Jong, E. M. G. J., Kimball, A. B., Langley, R. G., Lakdawala, N., Goyal, K., Lawson, F., Langholff, W., Hopkins, L., Fakharzadeh, S., Srivastava, B., \& Menter, A. (2018). Depressive symptoms, depression, and the effect of biologic therapy among patients in psoriasis longitudinal assessment and registry (PSOLAR). Journal of American Academy of Dermatology, 78(1), 70-80. https://doi.org/10.1016/j.jaad.2017. 08.051

16. Tee, S. I., Lim, Z. V., Theng, C. T., Chan, K. L., \& Giam, Y. C. (2016). A prospective cross-sectional study of anxiety and depression in patients with psoriasis in Singapore. Journal of the European Academy of Dermatology and Venereology, 30(7), 1159-1164. https://doi.org/10.1111/jdv.13615

17. World Health Organization. (1948). Constitution of the world health organization. World Health Organization.

18. Picardi, A., Pasquini, P., Abeni, D., Fassone, G., Mazzotti, E., \& Fava, G. A. (2005). Psychosomatic assessment of skin diseases in clinical practice. Psychotherapy and Psychosomatics, 74(5), 315-322. https://doi.org/10.1159/000086323

19. Offidani, E., Del Basso, D., Prignago, F., \& Tomba, E. (2016). Discriminating the presence of psychological distress in patients suffering from psoriasis: An application of the clinimetric approach in dermatology. Acta Dermato Venereologica, 96(217), 69-73. https://doi.org/10.2340/00015555-2369

20. Schuster, B., Ziehfreund, S., Albrecht, H., Spinner, C. D., Biedermann, T., Peifer, C., et al. (2020). Happiness in dermatology: A holistic evaluation of the mental burden of skin diseases. Journal of the European Academy of Dermatology and Venereology, 34(6), 1331-1339. https://doi.org/10.1111/jdv.16146
21. Solovan, C., Marcu, M., \& Chiticariu, E. (2014). Life satisfaction and beliefs about self and the world in patients with psoriasis: A brief assessment. European Journal of Dermatology, 24(2), 242-247. https://doi.org/10.1684/ejd.2014.2295

22. Rzeszutek, M., Podkowa, K., Pieta, M., Pankowski, D., \& CyranStemplewska, S. (2021). Comparative study of life satisfaction among patients with psoriasis versus healthy comparison group: The explanatory role of body image and resource profiles. Quality of Life Research, 30(1), 181-191. https://doi.org/10.1007/ s11136-020-02621-3

23. Martin-Brufau, R., Romero-Brufau, S., Martin-Gorgojo, A., Brufau Redondo, C., Corbalan, J., \& Ulnik, J. (2015). Psoriasis lesions are associated with specific types of emotions. Emotional profile in psoriasis. European Journal of Dermatology, 25(4), 329-334. https://doi.org/10.1684/ejd.2015.2577

24. Reimus, J. L., Vingerhoets, A. J., Soons, P. H., \& Korstanje, M. J. (2007). Suffering in psoriasis patients: Its relation with illness severity and subjective well-being. International Journal of Dermatology, 46(10), 1042-1045. https://doi.org/10.1111/j. 1365-4632.2007.03191.x

25. Diener, E., Oishi, S., \& Lucas, R. E. (2009). Subjective wellbeing: The science of happiness and life satisfaction. In S. J. Lopez \& C. R. Synder (Eds.), The Oxford handbook of positive psychology (2nd ed.). Oxford University Press.

26. Zidane, M., Dressler, C., Gaskins, M., \& Nast, A. (2019). Decision-analytic modeling for time-effectiveness of the sequence of induction treatments for moderate to severe plaque psoriasis. JAMA Dermatology. https://doi.org/10.1001/jamadermatol. 2019.2941

27. Pereira, M. G., Brito, L., \& Smith, T. (2012). Dyadic adjustment, family coping, body image, quality of life and psychological morbidity in patients with psoriasis and their partners. International Journal of Behavioral Medicine, 19(3), 260-269. https://doi.org/10.1007/s12529-011-9174-5

28. Cummins, R. A. (2013). Measuring happiness and subjective well-being. In S. A. David, I. Boniwell, \& A. Conley Ayers (Eds.), The Oxford handbook of happiness. Oxford University Press.

29. Veenhoven, R. (2013). Notions of the good life. In S. A. David, I. Boniwell, \& A. Conley Ayers (Eds.), The Oxford handbook of happiness. Oxford University Press.

30. Diener, E. (1984). Subjective well-being. Psychological Bulletin, 95(3), 542-575.

31. Bahmer, J. A., Kuhl, J., \& Bahmer, F. A. (2007). How do personality systems interact in patients with psoriasis, atopic dermatitis and urticaria? Acta Dermato Venereologica, 87(4), 317-324. https://doi.org/10.2340/00015555-0246

32. Jankowiak, B., Sekmistrz, S., Kowalewska, B., Niczyporuk, W., \& Krajewska-Kulak, E. (2013). Satisfaction with life in a group of psoriasis patients. Postepy Dermatol Alergol, 30(2), 85-90. https://doi.org/10.5114/pdia.2013.34156

33. Dowlatshahi, E. A., Wakkee, M., Arends, L. R., \& Nijsten, T. (2014). The prevalence and odds of depressive symptoms and clinical depression in psoriasis patients: A systematic review and meta-analysis. The Journal of Investigative Dermatology, 134(6), 1542-1551. https://doi.org/10.1038/jid.2013.508

34. Lukmanji, A., Basmadjian, R. B., Vallerand, I. A., Patten, S. B., \& Tang, K. L. (2020). Risk of depression in patients with psoriatic disease: A systematic review and meta-analysis. Journal of Cutaneous Medicine and Surgery. https://doi.org/10.1177/12034 75420977477

35. World Health Organization. (2016). ICD-10: International statistical classification of diseases and related health problems: 10th revision (5th ed.). Word Health Organization. 
36. George, D., \& Mallery, P. (2003). SPSS for windows step by step: A simple guide and reference. 11.0 update (4th ed.). Allyn \& Bacon.

37. Diener, E., Wirtz, D., Tov, W., Kim-Prieto, C., Choi, D., Oishi, S., \& Biswas-Diener, R. (2010). New measures of well-being: Flourishing and positive and negative feelings. Social Indicators Research, 39(2), 143.

38. Rahm, T., Heise, E., \& Schuldt, M. (2017). Measuring the frequency of emotions-validation of the scale of positive and negative experience (SPANE) in Germany. PLoS ONE, 12(2), e0171288. https://doi.org/10.1371/journal.pone.0171288

39. Watson, D., Clark, L. A., \& Tellegen, A. (1988). Development and validation of brief measures of positive and negative affect: The PANAS scales. Journal of Personality and Social Psychology, 54(6), 1063-1070.

40. Diener, E., Emmons, R. A., Larsen, R. J., \& Griffin, S. (1985). The satisfaction with life scale. Journal of Personality Assessment, 49, 71-75.

41. Glaesmer, H., Grande, G., Braehler, E., \& Roth, M. (2011). The German version of the satisfaction with life scale (SWLS). European Journal of Psychological Assessment, 27(2), 127-132. https://doi.org/10.1027/1015-5759/a000058

42. European Social Survey. (2016). ESS round 8 source questionnaire. ESS ERIC Headquarters c/o City University London.

43. World Health Organization. (1998). Use of well-being measures in primary health care-The DepCare Projects.

44. Lowe, B., Spitzer, R. L., Grafe, K., Kroenke, K., Quenter, A., Zipfel, S., et al. (2004). Comparative validity of three screening questionnaires for DSM-IV depressive disorders and physicians? Diagnoses. Journal of Affective Disorders, 78(2), 131-140. https://doi.org/10.1016/s0165-0327(02)00237-9

45. Hinkle, D., Wiersma, W., \& Jurs, S. (2003). Applied statistics for the behavioral sciences (5th ed.). Houghton Mifflin Company.

46. Guadagnoli, E., \& Velicer, W. F. (1988). Relation of sample size to the stability of component patterns. Psychological Bulletin, 103(2), 265-275.

47. Horn, J. L. (1965). A rationale and test for the number of factors in factor analysis. Psychometrika, 30, 179-185. https://doi.org/10. 1007/BF02289447

48. Brähler, E., Mühlan, H., Albani, C., \& Schmidt, S. (2007). Teststatistische Prüfung und Normierung der deutschen Versionen des EUROHIS-QOL Lebensqualität-Index und des WHO-5 Wohlbefindens-Index. Diagnostica, 53(2), 83-96. https://doi.org/10.1026/ 0012-1924.53.2.83

49. Schnaudt, C. (2016). Country-specific, cumulative ESS data set for Germany 2002-2004 (ESSDE1-7e01). Retrieved from https:// www.europeansocialsurvey.org/about/country/germany/german data.html

50. Knief, U., \& Forstmeier, W. (2020). Violating the normality assumption may be the lesser of two evils. bioRxiv. https://doi. org/10.1101/498931

51. Shields, J. L. (1978). An Empirical Investigation of the Effect of Heteroscedasticity and Heterogeneity of Variance on the
Analysis of Covariance and the Johnson-Neyman Technique. Army research inst. for the behavioral and social sciences. Retrieved from https://apps.dtic.mil/dtic/tr/fulltext/u2/a058205. pdf

52. Rasch, D., \& Guiard, V. (2004). The robustness of parametric statistical methods. Psychology Science, 46(2), 175-208.

53. Lorenzo-Seva, U., \& Ferrando, P. J. (2006). FACTOR: A computer program to fit the exploratory factor analysis model. Behavior Research Methods, 38(1), 88-91. https://doi.org/10.3758/ bf03192753

54. Hays, R. D., \& Fayers, P. M. (2021). Overlap of depressive symptoms with health-related quality-of-life measures. PharmacoEconomics, 39(6), 627-630. https://doi.org/10.1007/ s40273-020-00972-w

55. Westerhof, G. J., \& Keyes, C. L. (2010). Mental illness and mental health: The two continua model across the lifespan. Journal of Adult Development, 17(2), 110-119. https://doi.org/10.1007/ s10804-009-9082-y

56. Gargiulo, R. A., \& Stokes, M. A. (2008). Subjective well-being as an indicator for clinical depression. Social Indicators Research, 92(3), 517-527. https://doi.org/10.1007/s11205-008-9301-0

57. Lukat, J., Margraf, J., Lutz, R., van der Veld, W. M., \& Becker, E. S. (2016). Psychometric properties of the positive mental health scale (PMH-scale). BMC Psychology, 4(1), 8. https://doi.org/10. 1186/s40359-016-0111-x

58. Tizek, L., Schielein, M. C., \& Zink, A. (2020). PeakPASI: A new measurement tool in psoriasis care. Journal of the American Academy of Dermatology. https://doi.org/10.1016/j.jaad.2020.08. 096

59. World Health Organization. (2010). People-centred care in lowand middle-income countries. WHO Press.

60. Fortune, D. G., Richards, H. L., Kirby, B., Bowcock, S., Main, C. J., \& Griffiths, C. E. (2002). A cognitive-behavioural symptom management programme as an adjunct in psoriasis therapy. British Journal of Dermatology, 146(3), 458-465. https://doi.org/10. 1046/j.1365-2133.2002.04622.x

61. Rousset, L., \& Halioua, B. (2018). Stress and psoriasis. International Journal of Dermatology, 57(10), 1165-1172. https://doi. org/10.1111/ijd.14032

62. Robles, T. F., Brooks, K. P., \& Pressman, S. D. (2009). Trait positive affect buffers the effects of acute stress on skin barrier recovery. Health Psychology, 28(3), 373-378. https://doi.org/10.1037/ a0014662

63. Steptoe, A., Dockray, S., \& Wardle, J. (2009). Positive affect and psychobiological processes relevant to health. Journal of Personality, 77(6), 1747-1776. https://doi.org/10.1111/j.1467-6494. 2009.00599.x

Publisher's Note Springer Nature remains neutral with regard to jurisdictional claims in published maps and institutional affiliations. 